\title{
Topology of Linked Defects in Condensed Matter
}

\author{
M. I. Monastyrsky and V. S. Retakh \\ Institute of Theoretical and Experimental Physics, SU-117259 Moscow, USSR
}

\begin{abstract}
The present paper contains a systematic study of several linked singularities in condensed matter. We introduce a hierarchy of conservation laws in terms of differential forms corresponding to a sequence of linking invariants so that we can distinguish nontrivial links possessing zero Gauss linking coefficients. We obtain a set of topological obstruction rules for links in nematics, cholesterics, and superfluid ${ }^{3} \mathrm{He}$ and ${ }^{4} \mathrm{He}$.
\end{abstract}

\section{Introduction}

Line defects or disclinations, which are made visible in liquid crystals by polarized light, have been known in condensed matter physics since the discovery of nematics by $\mathrm{F}$. Reinitzer and O. Lehmann in the late 80-ies of the last century (as to a modern presentation, and references, see $[1,2])$. In fact, the term nematic is a graphic description (the greek word $v \eta \mu \alpha$ ) of the phenomenon. G. Friedel in his classical paper [3] found a partial explanation for the nature of such singularities. However, their systematic study was undertaken only in the 70-ies. In this respect the following results are worth mentioning: the phenomena of linked singularities observed experimentally by Bouligand [4], the topological approach based on homotopy groups first suggested in the paper [5] and, next, the linking of two defects investigated later by Poenaru and Toulouse $[6,7]$, using the concept of supergraduation of homotopy groups (the Whitehead product). This approach was also used in [8].

The present paper contains a systematic study of several linked singularities. Here it is worthwhile to note that the relevant theory, from the topological point of view, is the familiar theory of links, but with a view to physical applications we should take into account new structures related to thermodynamical problems requiring the introduction of the order parameter.

Our main results are the following:

1. We introduce a hierarchy of conservation laws in terms of differential forms corresponding to a sequence of linking invariants so that we can distinguish nontrivial links possessing zero Gauss linking coefficient. 
2. We obtain a set of topological obstruction rules for links in nematics, cholesterics, and superfluids ${ }^{3} \mathrm{He}$ and ${ }^{4} \mathrm{He}$. In particular, we should like to point out the case of ${ }^{3} \mathrm{He}$. Let us recall that the order parameter of superfluid ${ }^{3} \mathrm{He}$ is a complex $3 \times 3$ matrix subject to constraints required by the choice of a superfluid phase, and the minimization of the magnetic energy which can be cast in the form $F_{H}=g_{h} A_{p i} A_{q i} H_{p} H_{q}$.

Next, if the matrix of the order parameter has three different eigenvalues, then under the conditions indicated above the spin part of the order parameter is degenerate, and consists of three interlinked curves corresponding to the eigenvalues of the matrix $A_{p a}$.

Another example of a link in superfluid ${ }^{3} \mathrm{He}$ is provided by vortices corresponding to the orbital part of the order parameter. It is worth noticing that the topology of the vortices is the same as that of singular lines in nematics.

The paper is arranged as follows. In Sect. 1 we indicate the mathematical setting for defects in condensed media and introduce the concepts of homotopical and isotopical links. In Sect. 2 the standard linking theory is introduced using the Milnor coefficients. In Sect. 3 the general theory of Sects. 1 and 2 is applied to physical systems: nematic, biaxial nematic, cholesteric, and superfluid ${ }^{3} \mathrm{He}$. All these systems are characterized by the fundamental group $\pi_{1}(V)$ of the order parameter $V$. The most difficult case is the biaxial nematics and cholesteric, for in these cases, $\pi_{1}(V)$ is nonabelian. In Sect. 4 invariants of links are constructed via differential forms. To this end special cohomology operations (Massey products) are used. In the conclusion some possible physical applications of our work are discussed. It should be noticed that the mathematical apparatus used in this article is highly nonconventional and its presentation is rather scattery (see e.g. the excellent book [10]). To make this paper as selfcontained as possible we included a short summary of the necessary topological facts.

We expect the approach worked out in this paper will be useful for physical problems in which the sophisticated structures of links, singular lines, surfaces, and $n$-dimensional structures are of primary importance.

\section{Links and Defects}

\subsection{Topological Nature of Defects ${ }^{1}$}

A set $\ell$ of closed non-self-intersecting curves $\ell_{1}, \ldots, \ell_{n}$ in $S^{3}$ or $R^{3}$ is called a link. Let us fix a point $*$ outside $\ell$. We shall call the $i^{\text {th }}$ meridian of the link $\ell$ a non-selfintersecting loop $m_{i}$, beginning at the point $*$, contained in the complement to the set formed by curves of the link, so that $\ell_{i}$ intersects some surface bounded by $m_{i}$ at precisely one point, while none of the other $\ell_{j}$ have any common points with this surface. Evidently, the loop $m_{i}$ can be chosen arbitrary narrow.

Let us consider a manifold $M$, the physical space of the system, and the manifold $V$ of the order parameter space. Let $\Phi$ be a continuous map of the complement of a subset $\Sigma$ in the manifold $M$ into the order parameter space. The set $\Sigma$ is called the defect of $\Phi$.

1 In this paper we reserve the term "map" for continuous maps only 
Definition 1.1. The defect $\Sigma$ is essential for $\Phi$ if there is no continuous extension of $\Phi$ to any small disk intersects $\Sigma$.

Let us suppose that $M=S^{3}$, and $\Sigma=\ell=\left(\ell_{1}, \ldots, \ell_{n}\right)$ is a link.

Definition 1.2. The link $\ell$ is called a meridional-essential (m-essential) defect if $\Phi\left(m_{i}\right)$ is not contractible to a point ${ }^{2}$ in $V$ for all $m_{i}(1 \leqq i \leqq n)$.

It should be noted that an m-essential defect is essential. In fact, suppose that $\Sigma=\ell$ is not an essential defect. Then on one of the curves $\ell_{j}$ of $\ell$, we can find, a point such that $\Phi$ can be extended to its neighbourhood. In this neighbourhood let us choose a meridian $m_{j}$ and a surface $g$ bounded by $m_{j}$ so as to have $\Phi$ defined on $g$. Consequently, the image of the surface $g: \Phi(g)$ is contractible and $\Sigma$ is not $\mathrm{m}$-essential. Thus we see that the $\mathrm{m}$-essential defect is essential. On the contrary for $M=S^{3}$ and $\Sigma=\ell$, any essential defect is m-essential in many physically important cases. A rigorous treatment of the subject requires the following definitions.

Definition 1.3. Consider a map $\Phi: M \backslash \Sigma \rightarrow V$. We define a map $\Phi^{\prime}: M \backslash \Sigma \rightarrow V$ homotopically close to $\Phi$ if:

i) $\Phi^{\prime}$ is homotopic to $\Phi$,

ii) $\Phi^{\prime}$ coincides with $\Phi$ outside a sufficiently small neighbourhood of one of meridians.

We denote the set of such maps $\left\{\Phi^{\prime}\right\}$ as $\operatorname{HC}(\Phi)$.

Definition 1.4. An essential defect $\Sigma$ of the map $\Phi$ is stable if $\Sigma$ is an essential defect for any $\Phi^{\prime} \in \mathrm{HC}(\Phi)$.

Theorem 1.5. Let $M=S^{3}$ and an essential defect related to the link $\ell=\Sigma$ be stable for the map $\Phi: S^{3} \backslash \Sigma \rightarrow V$. Then defect $\Sigma$ is m-essential.

Proof. Suppose that $\Sigma$ is not m-essential, i.e. there exists a curve $\ell_{j}$ in the link $\ell$ with contractible $\Phi\left(m_{j}\right)$. Let $\tilde{\Phi}$ be the restriction of $\Phi$ to $m_{j}$; there is an extension $\varphi$ of $\tilde{\Phi}$ to a surface $g$ bounded by $m_{j}$. It is easy to construct a map $\Phi^{\prime}$ of $g \cup\left(S^{3} \backslash \Sigma\right)$ extending $\varphi$ and homotopic to $\Phi$ on $S^{3} \backslash \Sigma^{\prime}$. We can choose $m_{j}$ so narrow that the restriction $\Phi^{\prime}$ on $S^{3} \backslash \Sigma$ belongs to $\mathrm{HC}(\Phi)$. Since $\ell_{j}$ intersects the surface $g$, the defect $\Sigma$ is not essential for $\Phi^{\prime}$. This circumstance contradicts the stability of $\Sigma$ and proves the theorem.

The arguments used above are useful for studying other properties of defects generated by links of curves. Let us recall the definition of the parallel to the loop $\ell_{j}$ [13]. An $i^{\text {th }}$ parallel $\bar{\ell}_{i}$ of $\ell$ with respect to the path $p_{i}$ obtained by traversing $p_{i}$ from * to a sufficiently narrow tubular neighbourhood of $\ell_{i}$ then traversing along $\ell_{i}$ with linking coefficient zero and finally returning to $*$ along $p_{i}$. The choice of $p_{i}$ is not essential for the definition. We suppose also that the parallel $\bar{\ell}_{i}$ is contracted in $S^{3} \backslash \ell_{i}$.

Definition 1.6. Let $\Phi: S^{3} \backslash \ell \rightarrow V$. The defect $\Sigma=\ell$ is called strongly essential for the map $\Phi$ if $\Sigma$ remains essential for any map $\Phi^{\prime}: S^{3} \backslash \ell \rightarrow V$ homotopic to $\Phi$.

Theorem 1.7. If $\Sigma=\ell$ is a strongly essential defect of the map $\Phi$ and the curve $\ell_{i}$ of a link $\ell$ cannot be separated from other curves by a homeotopic image of a two dimensional sphere, then $\Phi\left(\bar{\ell}_{i}\right)$ is not contractible in $V$.

2 Hereafter the term contractible means contractible to a point 


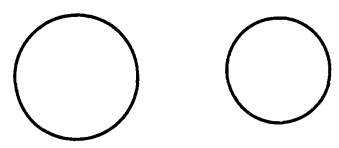

Fig. 1. Trivial link (disjoint circles $\ell_{1}$ and $\ell_{2}$ )



Fig. 2. Linked circles $k\left(\ell_{1}, \ell_{2}\right)=1$

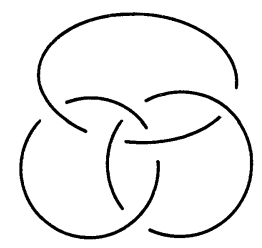

Fig. 3. Borromean rings

Proof. Let us suppose that $\Phi\left(\bar{\ell}_{i}\right)$ is contractible in $V$. Then the restriction $\Phi$ on $\bar{\ell}_{i}$ extends to a map $\psi$ of a surface $g$, bounded by $\ell_{i}$. Consider a homotopy of $\Phi$ into the map $\Phi^{\prime}$ which coincides with $\psi$ on the intersection $g \cap\left(S^{3} \backslash \Sigma\right)$. If the curve $\ell_{i}$ cannot be separated from the other curves, then at least one curve $\ell_{j}$ with $j \neq i$ intersects the surface $g$. It follows that $\Sigma$ is not an essential defect of the map $\Phi^{\prime}$, and by definition is not a strongly essential defect. This proves the theorem.

\subsection{Model Examples}

Let $\Phi: S^{3} \backslash \ell \rightarrow V$ be a map into the parameter order space with a defect $\Sigma$ formed by link $\ell$. Let us consider four model examples.

Example 1.8(Fig. 1). The link consists of two unlinked circles. In this case parallels of the link are contracted in $S^{3} \backslash \ell$ and hence, for any essential defect their images are contracted.

Example 1.9 (Fig. 2). The link is constructed by means of the simplest linking of two closed curves with the Gauss coefficient $\ell$. It is obvious that a parallel to one of the curves is homotopic to a meridian of the other one. Hence, in this case the m-essential defect results in the non-contracting of the parallel images in $V$.

Example 1.10 (Fig. 3). The link $\ell$ is a well known Borromean rings. It consists of three closed curves that can be split by cutting one of them. Here the parallel of one of the curves, e.g. $\bar{\ell}_{3}$, is homotopic to the commutator of the meridians of two others: $\bar{\ell}_{3} \sim\left[m_{1}, m_{2}\right]=m_{1} \cdot m_{2}, m_{1}^{-1} \cdot m_{2}^{-1}$. It is evident that when the fundamental group of the order parameter space is commutative the parallel images are 
Fig. 4. Whitehead's link

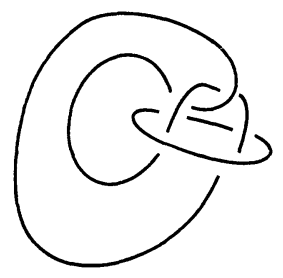

contracted in $V$. If, however, $\pi_{1}(V)$ is non-commutative then the situation can be more complicated.

For example, the fundamental group of a biaxial nematic or a cholesteric consists of 8 elements $\pm e_{1}, \pm e_{2}, \pm e_{3}, J, 1$ with relations $e_{1}^{2}=e_{2}^{2}=e_{3}^{2}=J, J^{2}=1$, $J e_{i}=e_{i} J=-e_{i}(i=1,2,3), e_{1} e_{2} e_{3}=J$. (For details see Subsect. 3.5 and [6].) The centre of this group consists of two elements $J$ and $\ell$. Therefore, if a defect is the Borromean rings and the images of all parallels and meridians are non-contractible in $V$, then the images of meridians $m_{1}, m_{2}, m_{3}$ are homotopic up to renumeration $\pm e_{1}, \pm e_{2}, \pm e_{3}$, and $\Phi\left(\bar{\ell}_{i}\right)$ is homotopic to $J$ for all $i$.

It is important that, although the Gauss linking coefficient for any two curves of the Borromean rings is zero, the properties of $\ell$, as it is easy to see, drastically differ from the three unlinked closed curves.

Example 1.11 (Fig. 4). Our last example is the well known Whitehead link $\ell$ of two curves $\ell_{1}$ and $\ell_{2}$. In this case the Gauss coefficient of the link $\ell$ is also equal to zero. But the topological properties of this link are considerably different from the trivial link in Example 1.8. The parallel $\ell_{2}$ in the Whitehead link is homotopic to the product of commutators of meridians $\left[m_{1}^{-1}, m_{2}\right] \circ\left[m_{1}, m_{2}\right]$. Consequently if $\pi_{1}(V)$ is commutative, $\Phi\left(\ell_{2}\right)$ is contractible in the case of any essential defect. It is worthwhile to note that $\Phi\left(\ell_{2}\right)$ is also contractible for biaxial nematic and cholesteric, even though $\pi_{1}(V)$ is not commutative.

It follows from the previous considerations that the topological properties of the map depend essentially on the structure of the defect. The knowledge of the Gauss coefficient is not sufficient for characterizing the structure of the defect. In the following sections we will construct the so-called higher linking coefficients, distinguishing between Borromean, Whitehead, and trivial links.

Some formulae for different homotopic classes of parallels in terms of homotopic classes of meridians have been given by Rolfsen [10] and Porter [11].

\section{General Theory of Links}

\subsection{Homotopic and Isotopic Properties of Links}

Two links $\ell=\left(\ell_{1}, \ldots, \ell_{n}\right)$ and $\ell^{\prime}=\left(\ell_{1}^{\prime}, \ldots, \ell_{n}^{\prime}\right)$ are called homotopic if there is a $\ell$-parameter family of maps $h_{t}$ from the space $C(n)$ consisting of $n$ disjoint oriented circles in the three-dimensional sphere $S^{\mathbf{3}}$ such that for each $t$ the disjoint circles in $C(n)$ have disjoint images in $S^{3}, h_{0}(C(n))=\ell, h_{1}(C(n))=\ell^{\prime}$. The curve $\ell_{i}$ is homotopically inlinkable with other components of $\ell$ if $\ell$ is homotopic to a link $\ell^{\prime}$ with $\ell_{i}^{\prime}$ contractible to a point. 
Two links $\ell=\left(\ell_{1}, \ldots, \ell_{n}\right)$ and $\ell^{\prime}=\left(\ell_{1}^{\prime}, \ldots, \ell_{n}^{\prime}\right)$ are called isotopic if there is a $\ell$-parameter family of maps $h_{t}$ from $C(n)$ to $S^{3}$ such that $h_{t}(C(n))$ is a link for any $t$, $h_{0}(C(n))=\ell, h_{1}(C(n))=\ell^{\prime}$. A curve $\ell_{i}$ is isotopically unlinkable with other components of $\ell$ if it can be separated by a homeomorphic image of the two dimensional sphere $S^{2}$ from other components.

A link $\ell=\left(\ell_{1}, \ldots, \ell_{n}\right)$ is homotopically trivial if it is homotopic to a link consisting of $n$ points. A link $\ell$ is isotopically trivial if its every component is isotopically unlinkable with the other ones.

From the physical point of view "unlinkability" of links means its isotopical "unlinkability." Homotopical unlinking allows self-intersection of a curve which "cost an energy." Homotopical "unlinkability" is rougher than the isotopical one.

Example 2.1. The Borromean rings are not homotopically trivial though each pair of it components is isotopically trivial.

Example 2.2. The Whitehead link is not isotopically trivial but it is homotopically trivial.

It is possible that, for a pair of two curves $\ell_{1}$ and $\ell_{2}, \ell_{1}$ is homotopically unlinked with $\ell_{2}$, but $\ell_{2}$ is not homotopically unlinked with $\ell_{1}$ (see [10, p. 69]).

The simplest linking invariant for two oriented closed curves $\ell_{1}, \ell_{2}$ in $S^{3}$ is the Gauss linking coefficient $k\left(\ell_{1}, \ell_{2}\right)$ [10] defined by the Gauss integral. For the simplest link on Fig. $2 k\left(\ell_{1}, \ell_{2}\right)=1$, and for the Whitehead link in Fig. 4 $k\left(\ell_{1}, \ell_{2}\right)=0$. Note, that $k\left(\ell_{1}, \ell_{2}\right)=0$ if $\ell_{1}$ and $\ell_{2}$ are homotopically unlinked. However, the Whitehead link shows that for isotopically linked curves this coefficient may be equal to zero. We shall describe in Subsect. 2.3 and Sect. 4 some higher order linking coefficients which allow us to solve the problem of homotopically and isotopically unlinking. The corresponding definitions are based on the properties of the Gauss coefficients of a link $\ell$ in $S^{3}$ [10].

\subsection{The First Order Milnor Coefficients}

Let $\ell=\left(\ell_{1}, \ldots, \ell_{n}\right)$ be a link in $S^{3}$. For this special case let us choose a point $*$ in the complement to $\ell$. Let us explain the meaning in which the homotopy classes of meridians are generators of the group $\pi_{1}\left(S^{3} \backslash \ell\right)$. To be more exact we assume every curve to be oriented. The orientation of a curve determines the orientation of its parallel and its meridian. The parallel inherits the orientation of the curve and the meridian $m_{i}$ is so oriented that $k\left(\ell_{i}, m_{i}\right)=1$.

Let us consider for an arbitrary group a lower central series (LCS) of subgroups $G_{1} \supset G_{2} \supset G_{3} \supset \ldots$ determined by the following rule: $G_{1}=G$ and $G_{n+1}$ is a subgroup of $G$ generated by the products $x y x^{-1} y^{-1}$, where $x \in G, y \in G_{n}$. The minimal $s$, for which $G_{s}$ consists of the unit element only, represents the measure of noncommutativity of the group $G$. If such $s$ exists the descending chain condition is fulfilled. In particular, for a commutative group such $s$ is equal to 2. For the fundamental group of the order parameter space of a biaxial nematic the corresponding $s$ equals 3. It should be noted that all $G_{i}$ are normal subgroups of $G$.

For $G=\pi_{1}\left(S^{3} \backslash \ell\right)$ the homotopical classes of meridians $m_{1}, \ldots, m_{n}$ are generators of the factor-group $G / G_{s}$ for $s \geqq 1$. Strictly speaking, the following proposition is valid. 
Proposition (Milnor's theorem [11]). For any $q$ there exists such a map $\alpha$ of a free group $F$ of noncommuting variables $x_{1}, \ldots, x_{n}$ on $G=\pi_{1}\left(S^{3} \backslash \ell\right)$ that:

i) $\alpha\left(X_{i}\right)$ is equal to the homotopy class of $m_{i}$;

ii) $\alpha$ induces isomorphism between $F / F_{q}$ and $G / G_{q}$;

iii) if $\alpha\left(y_{i}\right)$ is equal to the homotopy class of a parallel $\bar{\ell}_{i}$, then $\left[x_{i}, y_{i}\right]=1$.

Let us determine the Milnor coefficient. Let $\ell=\left(\ell_{1}, \ldots, \ell_{n}\right)$ be a link in $S^{3}$. Choose $q=n$ in the Milnor theorem. Let $\mu(i, j)$ be the sum of powers of the generators $x_{i}$ of the group $F$ in the decomposition of the elements $y_{j}$ for $i \neq j$. For example, for the Borromean rings we have $\mu(1,3)=\mu(2,3)=1-1=0$. The numbers $\mu(i, j)$ are called Milnor coefficients of the first order for the link $\ell$. They do not depend on the choice of parallels and meridians. Milnor shows [12] that $\mu(i, j)$ $=k(i, j)$. We recommend that the reader check the theorem by model examples. It is shown [12] that for a link of two curves $\ell_{1}$ and $\ell_{2}$ the equality $\mu(1,2)=0$ is necessary and sufficient for its homotopic unlinking. The Whitehead link shows that for isotopical unlinking this condition is not sufficient.

\subsection{The Higher Order Milnor Coefficients}

Let $\ell=\left(\ell_{1}, \ldots, \ell_{n}\right)$ - be a link in $S^{3}$. The first order Milnor coefficients, introduced in the previous subsection are determined by the representation of homotopic classes of parallels through homotopic classes of meridians. They are, however, very rough characteristics of such representations. Indeed, in the case of twocomponent links the formula $\mu(1,2)=0$ is valid for the trivial link and the Whitehead link, which is isotopically unlinked. For the Borromean rings $\ell=\left(\ell_{1}, \ell_{2}, \ell_{3}\right), \mu(i, j)=0$ for $1 \leqq i, j \leqq 3$, but this link is not trivial even in the homotopical sense. In order to distinguish between such links Milnor $[12,13]$ introduced higher order linking coefficients.

In the terminology of the previous subsection, we construct a homomorphism $\theta$ of the free group $F$ with generators $x_{1}, \ldots, x_{n}$ into the multiplicative group of formal power series with integral coefficients of concommuting variables $x_{1}, \ldots, x_{n}$ whose constant term is $\ell$. Put $\theta\left(x_{i}\right)=1+X_{i}$. It is evident that $\theta\left(x_{i}^{-1}\right)$ $=1-X_{i}+X_{i}^{2}-\ldots$ for $1 \leqq i \leqq n$. Let us determine the Milnor coefficient of the order $p-1$ for $p \geqq 2$. Consider the set of indices $1 \leqq i_{1}, \ldots, i_{p} \leqq n$. Let $y_{i p}$ be element transformed into the class of the parallel $\ell_{i p}$. We denote $\mu\left(i_{1}, \ldots, i_{p}\right)$ the coefficient of the monomial $X_{i_{1}} X_{i_{2}} \ldots X_{i_{p-1}}$ in the formal series $\theta\left(y_{i_{p}}\right)$. This is the $(p-1)^{\text {th }}$ Milnor coefficient. It is evident that the way of determining $\mu\left(i_{1}, i_{2}\right)$ presented in this subsection and in the previous one coincide. Now we shall present the values of the second and the third order Milnor coefficients for our model examples. For the Borromean rings $\mu(1,2,3)=1, \mu(2,1,3)=-1$. For the Whitehead link $\mu(1,2,2,1)$ $=\mu(1,1,2,2)=1, \mu(1,2,1,2)=-2, \ldots[11]$.

Milnor $[12,13]$ has shown that the numbers $\mu\left(i_{1}, \ldots, i_{p}\right)$ are uniquely determined modulo the number $\mu\left(j_{1}, \ldots, j_{q}\right)$, where $\left(j_{1}, \ldots, j_{q}\right)$ is an arbitrary set of numbers from the set $\left(i_{1}, \ldots, i_{p}\right)$ for $q<p$. In particular, if $\mu\left(j_{1}, \ldots, j_{q}\right)=0$ for all such sets, the number $\mu\left(i_{1}, \ldots, i_{p}\right)$ is determined uniquely. In the general case we consider the image $\mu\left(i_{1}, \ldots, i_{p}\right)$ in the group $\mathbb{Z} / \mu \mathbb{Z}$. Here $\mu$ is the maximal common divisor of the numbers $\mu\left(j_{1}, \ldots, j_{q}\right)$ (here $\left.q \leqq p-1\right)$. This image is denoted by $\bar{\mu}\left(i_{1}, \ldots, i_{p}\right)$ and is called the Milnor coefficient of the order $p-1$ for the link $\ell$. 
The Milnor coefficients $\bar{\mu}\left(i_{1}, \ldots, i_{p}\right)$ are homotopy invariants of a link in the case where there are no equal numbers in the set $\left(i_{1}, \ldots, i_{p}\right)$. In the opposite case the coefficients $\bar{\mu}\left(i_{1}, \ldots, i_{p}\right)$ are only isotopy invariants. Moreover, the link $\ell=\left(\ell_{1}, \ldots, \ell_{n}\right)$ is homotopically unlinked iff all $\bar{\mu}\left(i_{1}, \ldots, i_{p}\right)=0$ for $p \leqq n$ when there are no equal numbers in the set $\left(i_{1}, \ldots, i_{p}\right)$. Therefore, for the homotopical unlinking it is sufficient to examine the vanishing only of a finite set of numerical invariants.

A different situation arises in the isotopical case. It is evident that for isotopically trivial link $\ell \bar{\mu}\left(i_{1}, \ldots, i_{p}\right)=0$ for any set $\left(i_{1}, \ldots, i_{p}\right)$. The converse is not true, in general [13].

It is worthwhile to note that there exists a certain interrelation between the Milnor coefficients. The simplest one is the following:

$$
\bar{\mu}\left(i_{1}, \ldots, i_{p}\right)=(-1)^{p} \bar{\mu}\left(i_{p}, \ldots, i_{1}\right) .
$$

For other relations see $[12,13]$.

It is worthwhile to note that for a link of two curves the vanishing of the first order Milnor coefficient implies the vanishing of the second one. This fact is illustrated by the Whitehead link. Massey appears to have noticed the circumstance in the paper [14] which, regretfully, has not been available to the authors.

\section{The Homotopy Properties of Maps into the Parameter Order Spaces}

In this section we consider a map $\Phi: S^{3} \backslash \Sigma \rightarrow V$ with the defect $\Sigma$ which is equal to the link $\ell=\left(\ell_{1}, \ldots, \ell_{n}\right)$. We study the homotopy classes of the images $\Phi\left(\bar{\ell}_{i}\right)$, where $\bar{\ell}_{i}$ is a parallel of $\ell_{i}$, in connection with the properties of $\Phi\left(m_{i}\right)$, where $m_{i}$ is a meridian of $\ell_{i}$, using the Milnor coefficients. To obtain more specific information about these properties, we restrict the class of the order parameter spaces. Let us denote the basic point of $V$ by $\Phi(*)$.

\subsection{The Properties of $\Phi$ for the Finite Lower Central Series}

Let us consider the LCS of $\pi_{1}(V)$. The constraint for LCS to be finite is valid for all known order parameter spaces. For such spaces we have the following result.

Theorem 3.1. a) The homotopy classes of $\Phi\left(m_{i}\right)$ and $\Phi\left(\bar{\ell}_{i}\right)$ commute for every $1 \leqq i \leqq n$.

b) If every $\Phi\left(m_{i}\right)$ can be contracted in $V$ then every $\Phi\left(\bar{\ell}_{i}\right)$ can also be contracted.

c) If every Milnor coefficient for $\ell$ is equal to zero then $\Phi\left(\bar{\ell}_{i}\right)$ can be contracted in $V$ for $1 \leqq i \leqq n$.

Proof. The Milnor theorem from Subsect. 2.2 immediately implies the condition a).

If the parallel $\bar{\ell}_{i}$ can be contracted in $S^{3} \backslash \ell$ then $\Phi\left(\bar{\ell}_{i}\right)$ can be contracted in $V$. If $\ell_{i}$ cannot be contracted in $S^{3} \backslash \ell$, then the Milnor theorem implies that in $\pi_{1}(V)$ the homotopy class of $\Phi\left(\bar{\ell}_{i}\right)$ is equal to a product of homotopy classes of the images of meridians. So, if every $\Phi\left(m_{j}\right)$ can be contracted in $V$, the same is true for every $\Phi\left(\bar{\ell}_{i}\right)$. This proves $b$ ). 
The proof of c) is based on Subsect. 2.3.

In the following section we mention the connections between the values of linking coefficients and the properties of some physical systems and their order parameter spaces $V$. We consider the following examples:

1. $\pi_{1}(V)=\mathbb{Z}$. The considered physical system is superfluid ${ }^{4} \mathrm{He}$. Here the links consist of Abrikosov vortices.

2. $\pi_{1}(V)=\mathbb{Z}_{2}$. The physical system is nematic, $V=\mathrm{RP}^{2}$ i.e. the real projective plane. The order parameter space $V$ for the superfluid $A$-phase for ${ }^{3} \mathrm{He}$ under the spin orbital interaction $F_{a}$ has the same fundamental group. Here $V=\mathrm{SO}(3)$.

3. $\pi_{1}(V)=\mathbb{Z}_{4}$. The corresponding physical system is $A$-phase of ${ }^{3} \mathrm{He}$, $V=S^{2} \times \mathrm{SO}(3) / \mathbb{Z}_{2}$.

4. $\pi_{1}(V)$ equals to the group of quaternion units $Q$, and so $\pi_{1}(V)$ is noncommutative. In this case the corresponding physical systems are biaxial nematic and cholesteric and $V=\mathrm{SO}(3) / \mathscr{D}_{2}$ where $\mathscr{D}_{2}$ is the diedral group.

The identification of the order parameter space in these examples was obtained by many authors. The details can be found in the reviews $[16,17]$ as well as in the Poenaru lectures [18].

\section{2. $\pi_{1}(V)$ is Commutative}

Theorem 3.2. Fix $1 \leqq i \leqq n$. If $\mu(i, j)=k\left(\ell_{i}, \ell_{j}\right)=0$ for $1 \leqq j \leqq n, i \neq j$, then the image of the parallel $\bar{\ell}_{i}$ can be contracted in $V$.

Proof. Let $G=\pi_{1}\left(S^{3} \backslash \ell\right)$. The homotopy class of $\bar{\ell}_{i}$ equals some product $\Pi$ of homotopy classes of meridians in the factor group $G / G_{n}$. We can choose $\ell_{i}$ so that the sum of powers in which the homotopy class of $m_{i}$ entries in $\Pi$ equals to zero. The same is true for other meridians because $\mu(i, j)=0$ for every $j \neq i$. From the commutativity of $\pi_{1}(V)$ we conclude that the map induced by $\Phi$ sends the group $G_{n}$ to zero, so $\Phi\left(\overline{\ell_{i}}\right)$ is homotopic to a product of some powers of $\Phi\left(m_{j}\right), 1 \leqq j \leqq n$. The sum of powers in this product is zero, so $\Phi\left(\bar{\ell}_{i}\right)$ can be contracted in $V$ because of the commutativity of $\pi_{1}(V)$.

\section{3. $\pi_{1}(V)=\mathbb{Z}_{2}$}

In this case we can strengthen Theorem 3.2.

Theorem 3.3. Fix $1 \leqq i \leqq n$. Let the defect $\Sigma$ be m-essential. The loop $\Phi\left(\bar{\ell}_{i}\right)$ can be contracted in $V$ iff

$$
\sum_{j=1}^{i-1} \mu(i, j)+\sum_{j=i+1}^{n} \mu(i, j) \equiv 0(\bmod 2) .
$$

Proof. As in Subsect. 3.2 we can show that $\Phi\left(\bar{\ell}_{i}\right)$ is homotopic to the product

$$
\Phi\left(m_{1}\right)^{\mu(i, 1)} \ldots \Phi\left(m_{n}\right)^{\mu(i, n)},
$$

where $\mu(i, i)=0$.

Let $t$ be a loop in $V$ whose homotopy class is a generator in $\pi_{1}(V)$. Let $s=\sum_{j=1}^{n} \mu(i, j)$. Of course, $\Phi\left(m_{i}\right)$ is homotopic to $t$ for every $i$, because the defect is 
m-essential. So $\Phi\left(\bar{\ell}_{i}\right)$ is homotopic to $t^{s}$ and the loop $t^{s}$ can be contracted iff $s \equiv 0 \bmod 2$.

Example 3.4. Let us illustrate the theorem for $\Sigma=\ell=\left(\ell_{1}, \ell_{2}\right)$. If $\Phi\left(\bar{\ell}_{1}\right)$ can be contracted in $V$ then the linking coefficient $k\left(\ell_{1}, \ell_{2}\right)$ is even. If $k\left(\ell_{1}, \ell_{2}\right) \leqq 1$ for some energetic reasons (only such links appeared in the Bouligand experiments [4]) then $k\left(\ell_{1}, \ell_{2}\right)=0$, so $\ell_{1}$ and $\ell_{2}$ are homotopically unlinked.

\section{4. $\pi_{1}(V)=\mathbb{Z}_{4}$}

We have the following modification of Theorem 3.3.

Theorem 3.4. Let $t$ be a loop in $V$ whose homotopy class is a generator in $\pi_{1}(V)$. Let $\Phi\left(m_{j}\right)$ for every $j$ be homotopic to the loop $t^{s(j)}$, where $0 \leqq s(j) \leqq 3$. Then $\Phi\left(\bar{\ell}_{i}\right)$ can be contracted in $V$ iff

$$
\sum_{j=1}^{n} s(j) \cdot \mu(i, j) \equiv 0 \bmod 4
$$

\section{5. $V$ is the Order Parameter Space for Biaxial Nematic and Cholesteric}

In this case, $\pi_{1}(V)$ is equal to the unit quaternion group $Q$, it has 8 elements $\pm e_{1}$, $\pm e_{2}, \pm e_{3}, J, 1$ and the relations $e_{i}^{2}=J, J^{2}=1, e_{i} J=J e_{i}=-e_{i}, e_{1} e_{2} e_{3}=J$ $(i=1,2,3)$.

It is evident that

i) $J$ commutes with every element of $Q$ and commutator of $\pi_{1}(V)$ has only two elements, 1 and $J$.

ii) $e_{i}\left(-e_{i}\right)=1$ for $i=1,2,3$.

iii) $e_{i} e_{j}=\left(-e_{j}\right) e_{i}$ for $i \neq j$.

iv) $g^{4}=1$ for every $g \in \pi_{1}(V)$.

By Theorem 3.1 we have for a map $\Phi: S^{3} \backslash \Sigma \rightarrow V$ with $\Sigma=\ell\left(\ell_{1}, \ldots, \ell_{n}\right)$ that $\Phi\left(m_{i}\right)$ and $\Phi\left(\bar{\ell}_{i}\right)$ homotopically commute in $V$ for every $i$. Consequently, if $\Phi\left(m_{i}\right)$ is homotopic to $\pm e_{k}$, then $\Phi\left(\bar{\ell}_{i}\right)$ may be homotopic to $\pm e_{k}, J$ or 1 only.

The property iii) implies that every $\Phi\left(\bar{\ell}_{i}\right)$ may be homotopic to $1, J$ or to the product

$$
\pm \prod_{j=1}^{n} \Phi\left(\bar{\ell}_{j}\right)^{\mu(i, j)}
$$

This implies the following result.

Theorem 3.5. Let $\ell=\left(\ell_{1}, \ell_{2}\right)$ and the defect $\Sigma=\ell$ be m-essential. Denote by $h$ the homotopy class of $\Phi\left(m_{1}\right)$, then $\Phi\left(\bar{\ell}_{2}\right)$ can be contracted in $V$ iff :

$$
\begin{aligned}
& \mu(1,2) \equiv 0 \bmod 4 \text { when } h= \pm e_{i} \text { for some } i=1,2,3, \\
& \mu(1,2) \equiv 0 \bmod 2 \text { when } h=J .
\end{aligned}
$$

For the links of three circles we have

Theorem 3.6. Let $\Sigma=\ell=\left(\ell_{1}, \ell_{2}, \ell_{3}\right)$ and $\Phi\left(m_{1}\right)$ and $\Phi\left(m_{2}\right)$ do not homotopically commute in $V$. Then $\Phi\left(\bar{\ell}_{3}\right)$ can be contracted in $V$ iff :

$$
\mu(1,3)+\mu(2,3) \equiv 0 \bmod 4 \text {. }
$$




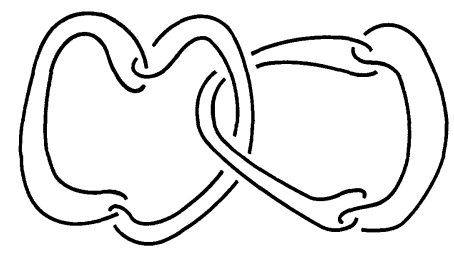

Fig. 5. Four component links

\section{The Differential Forms and the Higher Order Linking Coefficients}

In this section we define, using the differential forms, a lot of cohomological onstructions to the unlinking of a link $\ell=\left(\ell_{1}, \ldots, \ell_{n}\right)$ in $S^{3}$. V. Poenaru noted in his preprint "Superalgebras and confinement in condensed matter physics" that the Sullivan model [19] may be used for measuring the nontriviality of the Whitehead link $\ell=\left(\ell_{1}, \ell_{2}\right)$. Sullivan's model in this case is also based on studying the differential forms on $S^{3} \backslash \ell$. This construction includes the special graded differential skew-commutative algebra. On the contrary, our method is based on the straightforward calculation of differential forms on $S^{3} \backslash \ell$. We think that this method is simpler and more natural for physical applications.

The mentioned cohomological obstructions carry more information than the Alexander polynomials usually used by physicists [20]. For example, for the link on Fig. 5 the corresponding Alexander polynomial vanishes, but this link is homotopically unlinked. The Stallings conjecture, which was proved in [11] and [23] connect these cohomological obstructions with higher order Milnor coefficients.

Let $\ell=\left(\ell_{1}, \ell_{2}\right)$. Consider a surface $S_{2}$ bounded by $\ell_{2}$. It is well known [10] that the intersection index for $\ell_{1}$ and $S_{2}$ equals the Gauss linking coefficient $k\left(\ell_{1}, \ell_{2}\right)$. We use this definition of $k\left(\ell_{1}, \ell_{2}\right)$ to connect it with differential forms.

Let $u_{i}$ be a differential 1 -form which is the Alexander dual of the circle $\ell_{i}$. This form is defined on $S^{3} \backslash \ell_{i}$, it is closed and

$$
\int_{c} u_{i}=k\left(c, \ell_{i}\right)
$$

for any closed curve $c$ from the $S^{3} \backslash \ell_{i}$. (This is the characteristic property of $u_{i}$.) The cohomology class of $u_{i}$ is determined uniquely.

Let $B_{i}, i=1,2$ be the boundary of some tubular neighbourhood of $\ell_{i}$. Suppose that $B_{i}$ does not intersect the other circle. Then ${ }^{3}$

$$
\int_{B_{1}} u_{1} u_{2}=-\int_{B_{2}} u_{1} u_{2}=k\left(\ell_{1}, \ell_{2}\right) .
$$

We need the cohomology with compact support for determination of $k\left(\ell_{1}, \ell_{2}\right)$ using the integration of densities on $S^{3}$.

Let $M^{n}$ be a manifold and $\omega$ a differential form on $M^{n}$. The support of $\omega$ is the maximal closed subset of $M^{n}$ on which $\omega \neq 0$. If $M^{n}$ is compact then the support of any form on $M^{n}$ is compact. The cohomologies with compact support may be

3 We use the notation $u v$ for the skew product of differential forms 
defined by the DeRham complex of differential forms with compact support. For the curve $\ell_{i}$ we consider the Poincare dual cohomology class with compact support on $S^{\mathbf{3}} \backslash \ell_{i}$. This class is uniquely determined by a closed 2-form $v_{i}$ on $S^{\mathbf{3}} \backslash \ell_{i}$ such that

$$
\int_{z} v_{i}=\text { intersection index of } z \text { and } \ell_{i}
$$

for any 2-cycle $z$ in $S^{3} \backslash \ell_{i}$.

The 3 -forms $u_{1} v_{2}$ and $v_{1} u_{2}$ are defined on the whole $S^{3}$ and

$$
\int_{S^{3}} u_{1} v_{2}=-\int_{S^{3}} v_{1} u_{2}=k\left(\ell_{1}, \ell_{2}\right) \text {. }
$$

The coefficients $k\left(\ell_{i}, \ell_{j}\right)$ present some number invariants of the link $\ell=\left(\ell_{1}, \ldots, \ell_{n}\right)$. We can also denote the single first order linking coefficient of $\ell$ by the formula

$$
k(\ell)=\max _{\ell \leqq i \leqq j \leqq n}\left|k\left(\ell_{i}, \ell_{j}\right)\right| .
$$

If $\ell$ is isotopically unlinked then $\bar{k}(\ell)=0$. However, for links in Figs. $3-5$ this number is also zero but such links cannot be unlinked. We need high order linking coefficients for the description of such links.

Note that $k\left(\ell_{1}, \ell_{2}\right)=0$ implies that there is a 1 -form $u_{12}$ on $S^{3} \backslash \ell$ and 2-forms $v_{12}, v_{12}^{\prime}$ with compact supports on $S^{3}$ such that

$$
d u_{12}=u_{1} u_{2}, \quad d v_{12}=-v_{1} u_{2}, \quad d v_{12}^{\prime}=u_{1} v_{2} .
$$

Let $\bar{k}(\ell)=0$ for $\ell=\left(\ell_{1}, \ell_{2}, \ell_{3}\right)$. Define the Alexander dual 1 -form $u_{i}$ and the Poincaré 2 -forms $v_{i j}$ with compact supports $(1 \leqq i<j \leqq 3)$. It is easy to check that the forms

$$
\begin{aligned}
& \tilde{u}_{123}=u_{12} u_{3}+u_{1} u_{23}, \\
& \tilde{v}_{123}=-v_{12} u_{3}+v_{1} u_{23}, \\
& \tilde{v}_{123}^{\prime}=u_{12} v_{3}+u_{1} v_{23}
\end{aligned}
$$

are closed. The form $u_{123}$ is defined on $S^{3} \backslash \ell$. We can choose $v_{12}$ and $v_{23}$ so that $v_{123}$ and $v_{123}^{\prime}$ are defined on the whole $S^{3}$.

The form $\tilde{u}_{123}$ defines the cohomology class in $H^{2}\left(S^{3} \backslash \ell\right)$ and the forms $\tilde{v}_{123}$, $\tilde{v}_{123}^{\prime}$ define the cohomology classes in $H^{3}\left(S^{3}\right)$. These classes are called the Massey products of the cohomology classes of $u_{1}, u_{2}, u_{3} ; v_{1}, u_{2}, u_{3}$ and $u_{1}, u_{2}, v_{3}$, respectively $[15,25]$. They depend on the cohomology classes of $u_{1}, \ldots, v_{3}$ only. We denote them by $\left\langle\operatorname{cl} u_{1}, \operatorname{cl} u_{2}, \operatorname{cl} u_{3}\right\rangle, \ldots$ where $\operatorname{cl} \omega$ is the cohomology class of $\omega$.

One can show that there exists an integer $k_{2}(\ell)$ such that:

$$
\int_{B_{1}} \tilde{u}_{123}=-\int_{B_{3}} \tilde{u}_{123}=\int_{S^{3}} \tilde{v}_{123}=\int_{S^{3}} \tilde{v}_{123}^{\prime}=k_{2}(\ell) .
$$

This integer does not depend on the choice of $u_{12}, u_{23}, v_{12}$, and $v_{23}$. This is a linking coefficient of the second order.

For the link $\ell=\left(\ell_{1}, \ldots, \ell_{n}\right), n \geqq 3$ we consider the sublinks $\ell_{i j k}=\left(\ell_{i}, \ell_{j}, \ell_{k}\right)$ for $1 \leqq i<j<k \leqq n$. It is possible to define the linking coefficient of the second order $\bar{k}_{2}(\ell)$ by the formula

$$
\bar{k}_{2}(\ell)=\max _{\ell \leqq i<j<k \leqq n}\left|k_{2}\left(\ell_{i j k}\right)\right|
$$


For the Borromean rings this coefficient is equal to 1 . If $\ell$ is homotopically unlinked then $k_{2}(\ell)=0$. But for the link in Fig. $5 k_{2}(\ell)=0$, although this link is not homotopically unlinked. We introduce the linking coefficient of the third order which is not equal to zero for this link.

Let $\ell=\left(\ell_{1}, \ell_{2}, \ell_{3}, \ell_{4}\right)$ and $k_{2}(\ell)=0$. There exist 1 -forms $u_{123}, u_{234}$ in $S^{3} \backslash \ell_{123}$ and $S^{3} \backslash \ell_{234}$, respectively such that $d u_{123}=\tilde{u}_{123}, d u_{234}=\tilde{u}_{234}$. There also exist 2-forms $v_{123}, v_{234}$ with compact supports such that their supports do not intersect $\ell_{1}$ and $\ell_{4}$, respectively and $d v_{123}=\tilde{v}_{123}, d v_{234}=\tilde{v}_{234}$. We can choose $v_{123}$ and $v_{234}$ so that $\tilde{v}_{1234}=u_{1} v_{234}+u_{12} v_{34}+u_{123} u_{4}$ and $\tilde{v}_{1234}^{\prime}=-v_{1} u_{234}+v_{12} u_{34}+v_{123} u_{4}$ are defined on the whole $S^{3}$. It is easy to check that $\tilde{v}_{1234}$ and $\tilde{v}_{1234}^{\prime}$ are closed. They define cohomology classes in $H^{3}\left(S^{3}\right)$. The 2-form $\tilde{u}_{1234}=u_{1} u_{234}+u_{12} u_{34}+u_{123} u_{4}$ defines the class in $H^{2}\left(S^{3} \backslash \ell\right)$. One calls the defined classes the Massey products $\left\langle\operatorname{cl} v_{1}, \operatorname{cl} u_{2}, \operatorname{cl} u_{3}, \operatorname{cl} u_{4}\right\rangle, \ldots$, respectively. They do not depend on the cohomology classes of $u_{1}, \ldots, v_{4}$.

One can show that there exists an integer $k_{3}(\ell)$ such that

$$
k_{3}(\ell)=\int_{B_{\ell}} \tilde{u}_{1234}=\ldots=\int_{S^{3}} \tilde{v}_{1234}^{\prime} .
$$

This integer does not depend on the choice $u_{12}, u_{23}, u_{123}, \ldots$.

For the link in Fig. $5 k_{3}(\ell)=1$ [22]. Note that the value of $k_{3}(\ell)$ for $\ell=\left(\ell_{1}, \ell_{2}, \ell_{3}, \ell_{4}\right)$ does not change under the cyclic permutation of $(1,2,3,4)$, but may change under other permutations (see [22]). We can also define the third degree order linking coefficient for two closed curves. From the geometric point of view we consider several images of the same curve under small translations. For example, we consider $\ell_{1222}=\left(\ell_{1}, \ell_{2}, \ell_{2}, \ell_{2}\right)$ instead of $\ell_{12}=\left(\ell_{1}, \ell_{2}\right)$. From the algebraic point of view in this case $u_{2}=u_{3}=u_{4}$ and $u_{2} u_{3}=u_{3} u_{4}=0, u_{23}=u_{34}=0$. The Whitehead link $\ell=\left(\ell_{1}, \ell_{2}\right)$ may be transformed to $\ell_{1212}=\left(\ell_{1}, \ell_{2}, \ell_{1}, \ell_{2}\right)$ with $u_{3}=u_{1}, u_{4}=u_{2}$ and so on. We have

$$
k_{3}\left(\ell_{1222}\right)=1, \quad k_{3}\left(\ell_{1212}\right)=-2 .
$$

Now let us define for the link $\ell=\left(\ell_{1}, \ldots, \ell_{n}\right)$ the $(p-1)^{\text {th }}$ order invariants $k\left(i_{1}, \ldots, i_{p}\right)$ using the curves $\ell_{i_{1}}, \ldots, \ell_{i_{p}}$, some of them may coincide with each other. Under this definition $k(\ell)=k(1, \ldots, n)$. The general $(p-1)^{\text {th }}$ linking order coefficient of $\ell$ may be defined by the formula

$$
\bar{k}_{p-1}(\ell)=\max _{1 \leqq i_{1}, \ldots, i_{p} \leqq n}\left|k\left(i_{1}, \ldots, i_{p}\right)\right| .
$$

We define $k\left(i_{1}, \ldots, i_{p}\right)$ using the $(p-1)^{\text {th }}$ order Massey product $[16,25]$.

Let $\omega_{1}, \ldots, \omega_{s}$ - be closed differential forms on the manifold $M, \operatorname{deg} \omega_{i}=\alpha_{i}$ and $\operatorname{cl} \omega_{i}$ is the cohomology class of $\omega_{i}$ for $1 \leqq i \leqq s$. The Massey product $\left\langle\operatorname{cl} \omega_{1}, \ldots, \operatorname{cl} \omega_{s}\right\rangle$ is defined if there exist differential forms $\omega_{i j}$ for $0 \leqq j-i<s-1$ such that

i) $\omega_{i i}=\omega_{i}$,

ii) $d \omega_{i j}=\sum_{\substack{k=i \\ k=j-1}}^{k=j-1} \tilde{\omega}_{i k} \omega_{k+1 j} ; \tilde{\omega}_{i k}=(-1)^{\operatorname{deg} \omega_{i k}+1} \omega_{i k}$.

The form $\sum_{k=1}^{k} \tilde{\omega}_{1 k} \omega_{k+1 s}$ is closed and its cohomology class is called the value of $\left\langle\operatorname{cl} \omega_{1}, \ldots, \operatorname{cl} \omega_{n}\right\rangle$. For simplicity we write $\left\langle\omega_{1}, \ldots, \omega_{n}\right\rangle$ instead of $\left\langle\operatorname{cl} \omega_{1}, \ldots, \operatorname{cl} \omega_{n}\right\rangle$. 
Note that the Massey product may have many values but they depend only on cohomology classes of $\omega_{i},(i=1,2, \ldots, n)$.

If we use the forms $\omega_{i}$ with compact supports then the forms $\omega_{i j}$ must have compact supports, too. In this case the values of Massey products are cohomology classes with compact supports (or usually cohomology classes if $M$ is compact).

We call the forms $\omega_{i j}$ for $i \neq j$ the intermediate forms.

For the link $\ell=\left(\ell_{1}, \ldots, \ell_{n}\right)$ in $S^{3}$ we consider the Massey product $\left\langle\omega_{i_{1}}, \ldots, \omega_{i_{p}}\right\rangle$ in the following cases.

1) $M=S^{3} \backslash \ell, \omega_{i_{p}}$ is equal to 1 -form $u_{i}$, which is the Alexander dual to a circle $\ell_{i}$ for $1 \leqq i_{p} \leqq n$.

2) $M=S^{3}, \omega_{i_{1}}$ is equal to 2-form $v_{i_{1}}$ with compact support which is the Poincaré dual to a circle $\ell_{i_{1}}$ and $\omega_{i_{p}}=\varphi_{i_{p}} \cdot u_{i_{p}}$, where $\varphi_{i_{p}}$ is a smooth function on $S^{3}$ and $\varphi_{i_{p}}=0$ in a small neighbourhood of $\ell_{i_{p}}, 2 \leqq i_{p} \leqq n$. In this case we use $u_{i_{p}}$ instead of $\varphi_{i_{p}} \cdot u_{i_{p}}$.

3) $M=S^{3}$, the forms are $u_{i_{1}}, \ldots, v_{i_{p}}$ by analogy with 2 ).

Theorem 4.1. Let $i_{1}=1, i_{p}=n$ and one of the three Massey products $\left\langle u_{i_{1}}, \ldots, u_{i_{p}}\right\rangle$, $\left\langle v_{i_{1}}, \ldots, u_{i_{p}}\right\rangle,\left\langle u_{i_{1}}, \ldots, v_{i_{p}}\right\rangle$ is defined. Then two other products are also defined and there exists an integer

$$
\begin{aligned}
k\left(i_{1}, \ldots, i_{p}\right) & =\int_{B_{i_{1}}}\left\langle u_{i_{1}}, \ldots, u_{i_{p}}\right\rangle=(-1)^{p} \int_{B_{i_{p}}}\left\langle u_{i_{1}}, \ldots, u_{i_{p}}\right\rangle \\
& =\int_{S^{3}}\left\langle v_{i_{1}}, \ldots, u_{i_{p}}\right\rangle=(-1)^{p} \int_{S^{3}} u_{i_{1}}, \ldots, v_{i_{p}} .
\end{aligned}
$$

This integer is the $(p-1)^{\text {th }}$ order linking coefficient of $\ell$. The $q^{\text {th }}$ order linking coefficient is defined iff every $r^{\text {th }}$ order linking coefficient vanishes for $r<q$.

The Stallings conjecture [21] which was proved by Turaev [23] and Porter [11] implies that $k\left(i_{1}, \ldots, i_{p}\right)=\mu\left(i_{1}, \ldots, i_{p}\right)$ if every $r^{\text {th }}$ order linking coefficient vanishes for $r \leqq p-1$. By [11] in this case $\left\langle u_{i_{1}}, \ldots, u_{i_{p}}\right\rangle=(-1)^{p} \mu\left(i_{1}, \ldots, i_{p}\right) \gamma$, where $\gamma$ is the Poincaré-Lefschetz dual to a path from $\ell_{i_{1}}$ to $\ell_{i_{p}}$.

There exists a more general statement $[11,23]$ that does not require that every $r^{\text {th }}$ order linking coefficient should vanish for $r \leqq p-1$.

\section{Conclusion}

In this paper a topological approach to the recognition of non-trivial links is developed. The physical applications are mainly illustrative in nature and consist in describing the restrictions on types of defect links in condensed matter. However, we hope that our method will be useful in other physical problems: for example, in the statistics of polymer chains or in the turbulence in superfluids. The influence of linked vortical threads on the turbulence origin in homogeneous superfluid ${ }^{4} \mathrm{He}$ was considered in [26]. Another example is the magnet hydrodynamics where the formulae for linking coefficients from Sect. 4 play the role of topological conservation laws for magnet power threads frozen in a field. greatly stimulated this article. In $[6,7] \mathrm{V}$. Poenaru and G. Toulouse study the properties of links of two line defects using the Whitehead product in the homotopy groups of order parameter spaces. One can get their results from our paper using the relationship between the Whitehead and 
Massey products and Milnor coefficients. We want to thank V. Golo, Y. Eidus, T. Samsonova, A. Sosinsky for their assistance in the final preparation of this text. We are very grateful to S.P. Novikov for helpful discussions and comments. We also would like to thank G. E. Volovik for his careful reading and correction of the text.

\section{References}

1. De Gennes, P.G.: The physics of liquid crystals. Oxford: Clarendon Press 1974

2. Chandrasekhar, S.: Liquid crystals. Cambridge: Cambridge University Press 1977

3. Friedel, G.: Les états mesomorphes de la matiere. Ann. Phys. 18, 273-274 (1922)

4. Bouligand, Y.: Recherches sur les textures des états mesomorphes 6. J. Phys. 35, 959-981 (1974)

5. Toulouse, G., Kleman, M.: Principles of a classification of defects in ordered media. J. Phys. Lett. 37, LI49 (1976)

6. Poenaru, V., Toulouse, G.: The crossing of defects in ordered media and the topology of 3-manifolds. J. Phys. (Paris) 38, 887-895 (1977)

7. Poenaru, V., Toulouse, G.: Topological solitons and graded Lie algebras. J. Math. Phys. 20 (1), 13-19 (1979)

8. Jänich, K., Trebin, H.-R.: Dissentanglement of line defects in ordered media. Balian, R. et al. (eds.). Les Houches, Session XXXV 1980 - Physique des Defauts/Physics of Defects. Amsterdam: North-Holland 1981

9. Golo, V.L., Monastyrsky, M.I.: Gauge group and phases of superfluid ${ }^{3} \mathrm{He}$. Lett. Math. Phys. 2, 373-378 (1978)

10. Rolfsen, D.: Knots and links. Wilmington, Publish or Perish 1976

11. Porter, R.: Milnor's $\bar{\mu}$ invariants and Massey products. Trans. Am. Math. Soc. 257 (1), 39-71 (1980)

12. Milnor, J.: Link groups. Ann. Math. 59, 177-195 (1954)

13. Milnor, J.: Isotopy of links. Algebraic geometry and topology. A Symposium in honor of S. Lefschetz, pp. 280-306. Princeton, NJ: Princeton University Press 1957

14. Massey, W.S.: Higher order linking numbers, 174-205. Conf. on Algebraic Topology. Chicago: University of Chicago 1968

15. Massey, W.S.: Symposium Int. de Topologia Algebraica, Mexico, 145, 1958

16. Legget, A.J.: A theoretical description of the new phases of liquid ${ }^{3} \mathrm{He}$. Rev. Mod. Phys. 47, 346-414 (1975)

17. Mermin, N.D.: The topological theory of defects in ordered media. Rev. Mod. Phys. 51, 591-648 (1979)

18. Poenaru, V.: Elementary algebraic topology related to the theory of defects and textures, p. 265. Les Houches 1978

19. Sullivan, D.: Infinitesimal calculations in topology. IHES Publ. Math. 4, 269-331 (1977)

20. Frank-Kamenetskii, M.D., Vologodskii, A.V.: Topological aspects of polymer physics: the theory and its biophysics applications. Usp. Phys. Nauk. 134 N 4, 641-673 (1981) (in Russian)

21. Stallings, J.: Homology and central series of groups. J. Algebra 2, 170-181 (1965)

22. O'Neil: Higher order Massey products. Trans. Am. Math. Soc. 248 (1), 37-66 (1979)

23. Turaev, V.G.: Milnor invariants and Massey products. Zap. Semin. Lomi 66, 189-203 (1976) (in Russian)

24. Milnor, J.W., Stasheff, J.D.: Characteristic classes. Princeton, NJ : Princeton University Press 1974

25. Kraines, D.: Massey higher products. Trans. Am. Math. Soc. 124, 431-449 (1966)

26. Schwarz, K.: Generation of superfluid turbulence deduced from simple dynamical rules. Phys. Rev. Lett. 49, 283-285 (1982)

Communicated by Ya. G. Sinai

Received March 15, 1985; in revised form July 1, 1985 
